\title{
MAPEAMENTO DO RISCO DE CONTAMINAÇÃO DAS ÁGUAS SUBTERRÂNEAS EM ZONAS URBANAS: proposta de um índice de perigo fuzzy-AHP
}

Erico Gaspar LISBOA ${ }^{1}$

Ronaldo Lopes Rodrigues MENDES²

Leonardo Augusto Lobato BELLO

\author{
${ }^{1}$ Doutorando em Engenharia Civil pela Faculdade de Engenharia da Universidade do Porto. dec12006@fe.up.pt \\ 2 Professor Associado I da Universidade Federal do Pará. rlopes@ufpa.br \\ 3 Professor Titular da Universidade da Amazônia. leonardo.bello@unama.br
}

Recebido em: 30/08/2016 - Aprovado em: 25/09/2016 - Disponibilizado em: 18/12/2016

\begin{abstract}
RESUMO
O objetivo desse trabalho é mapear o risco de contaminação das águas subterrâneas em zonas urbanas e periurbanas. Para tanto, recorreu-se a uma concepção de risco que é produto da associação entre a vulnerabilidade intrínseca e o perigo da fonte contaminante. Para avaliar a vulnerabilidade utilizou-se o método GOD. Em seguida, a partir do potencial gerador de nitrogênio-nitrato $\left(\mathrm{N}-\mathrm{NO}_{3}\right)$, estimado em função da densidade populacional e do potencial de $\mathrm{N}-\mathrm{NO}_{3}$ por habitante, quantificou-se o grau de periculosidade, por uma proposta de um modelo multi-criterial fuzzy-AHP. Ambas as avaliações produziram índices que foram interpolados por método geoestatístico, resultando em mapas da vulnerabilidade e perigo. A associação dos referidos mapas produziu-se o mapa do risco. A aplicação da metodologia proposta abrangeu a cidade de Belém, Pará, Brasil, em que se mapeou a vulnerabilidade de aquíferos freáticos, perigo e risco de contaminação da zona urbana e periurbana. Os resultados apresentaram-se como uma ferramenta de gestão ambiental com fulcro a proteção das águas subterrâneas.
\end{abstract}

Palavras-Chave: Risco de contaminação; Vulnerabilidade intrínseca; Perigo; modelo fuzzy-AHP.

\begin{abstract}
The aim of this study is to map the risk of contamination of groundwater in urban areas. To do so, it resorted to a risk of conception, which is the association product of the intrinsic vulnerability and the hazard of contaminating source. To assess the vulnerability used the GOD method. Then from nitrite-nitrate-generating potential $\left(\mathrm{N}-\mathrm{NO}_{3}\right)$, estimated on the basis of population density and the potential of $\mathrm{N}_{-} \mathrm{NO}_{3}$ per capita, we quantified the degree of hazard, by a proposal for a multicriterial fuzzy-AHP model. Both assessments produced indexes that were interpolated by geostatistical method, resulting in maps of vulnerability and danger. The association of these maps produced to the risk map. The application of the proposed methodology included the city of Belém, Pará, Brazil, which it mapped the vulnerability of groundwater aquifers, hazard and risk of contamination of urban area. The results were presented as an environmental management tool with core protection of groundwater.
\end{abstract}

Keywords: Risk of contamination; Intrinsic Vulnerability; Hazard, fuzzy-AHP model.

\section{Introdução}

A maioria dos países depende das águas subterrâneas para suprir as suas necessidades de abastecimento doméstico, agrícola e industrial. Contudo, a falta de um controle racional do uso e proteção é um cenário presente e preocupante. Uma dessas preocupações refere-se à ameaça da contaminação que podem comprometer a qualidade, e assim, gerar impactos de ordem ambiental e socioeconômica, inviabilizando a utilização da água para múltiplas finalidades.
As ameaças (ou perigo) podem transformar-se num evento de contaminação, geralmente desencadeado, quer por acidentes em atividades que manuseiam metais pesados, produtos tóxicos, elementos radioativos, hidrocarbonetos, entre outros, quer pela disposição final e má gestão de lixiviados de resíduos (fontes pontuais); e, também pelo uso indiscriminado de defensivos agrícolas em zonas rurais (fonte difusa). Dando ênfase as ameaças em zonas urbanas, a ausência e/ou 
ineficiência de ações estruturais e estruturantes dos sistemas de esgotamento sanitário podem contribuir, e se constituir, no fator de elevação das concentrações de nitratos $\left(\mathrm{NO}_{3}\right)$ nas águas subterrâneas. No Reino Unido, por exemplo, estudos realizados por Lerner et al. (1999) correlacionaram as altas concentrações desse composto, detectadas nas águas subterrâneas, ao crescimento da cidade de Nottingham. Whitehead et al. (1999), a partir das análises de isótopos de nitrogênio $\left({ }^{15} \mathrm{~N}\right)$, relevaram que a rede de esgoto, concebida no início do século XVIII, constitui-se como fonte de contaminação do principal aquífero da cidade de Liverpool.

Em Telavive, Israel, Zilberbrand et al. (2001) referiram que a contaminação das águas subterrâneas por $\mathrm{NO}_{3}$ advinham de fossas negras. No Brasil, Godoy (2004) conduziu pesquisas que associaram a contaminação por $\mathrm{NO}_{3}$ com o vazamento de esgoto na cidade de Presidente Prudente, estado de São Paulo.

A contaminação da água por $\mathrm{NO}_{3}$ iniciase pela decomposição da matéria orgânica, transformada e oxidada por reações químicas e biológicas, resultando na presença de $\mathrm{NO}_{3}$ no solo que, após um processo de mineralização, passará a forma de nitrogênio-nitrato $\left(\mathrm{N}-\mathrm{NO}_{3}\right)$. Como esse composto é solúvel na água, sua lixiviação pode contaminar rapidamente aquíferos superficiais. Assim, caso os aquíferos sejam contaminados, o consumo da água com uma concentração acima do valor máximo permitido (50 mg $\mathrm{N}-\mathrm{NO}_{3} / \mathrm{L}$ ), pode provocar efeitos adversos à saúde humana, como a indução à metahemoglobina e a formação potencial de nitrosaminas e nitrosamidas carcinogênicas, teratogênicas e mutagênicas (e.g. cânceres gástricos, linfomas nãoHodgkins, febre tifoide, cólera, diarreia, etc.

Portanto, analisar o risco de contaminação das águas subterrâneas em zonas urbanas, cujo potencial gerador de $\mathrm{N}-\mathrm{NO}_{3}$ pode apresentar-se como uma ameaça a saúde humana, além de ser uma ferramenta de gestão ambiental; fortalece-se a ideia de que a melhor opção é investir em ações preventivas, em face da dificuldade de remediar sítios já contaminados até níveis aceitáveis de potabilidade.

Desse modo, pode-se deliberar ações preventivas, a partir de tomadas de decições que possam diminuir o risco, tais como: maior abrangência da rede de esgotamento sanitário (medidas estruturais), e, inclusão da rede existente num plano de gestão de condutas (medidas estruturantes). Assim, a partir de uma concepção para avaliar o risco, propõem-se uma metodologia para analisar e mapear o risco de contaminação das águas subterrâneas.

Nesses termos, Wang et at. (2012) referiram sobre o risco básico de contaminação das águas subterrâneas, que pode ser analisado pela relação entre os graus de vulnerabilidade intrínseca de aquíferos e da periculosidade da fonte contaminante.

A avaliação da vulnerabilidade pode ser referida por métodos que funcionam como um tipo de sistema de indexação, estruturado da seguinte maneira: a vulnerabilidade intrínseca é 
representado por uma saída Y (índice), resultante de um processo que integra um conjunto de variáveis de entrada $\mathbf{X}=\left(\mathbf{x}_{\mathbf{1}}\right.$, $\left.\mathbf{x}_{2}, \ldots \mathbf{x}_{\mathbf{i}}\right)$. Por alguns tipos de métodos essas variáveis de entradas, que representam parâmetros hidrogeológicos, são ponderadas, e.g. pelo método DRASTIC (ALLER et al, 1987); ou não, e.g. pelo método GOD (FOSTER, HIRATA, 1988).

Para avaliar o perigo faz-se necessário, além de identificar agentes poluidores, quantificar cargas de um contaminante. Essa identificação é gerenciado pelo cadastramento de fontes de contaminação (U.S. EPA, 1991; ZAPOROZEC, 2001). A carga de um dado contaminante é dependente da sobrecarga hidráulica, concentração, mobilidade e persistência.

Nesses termos, Foster et al. (2002) propuseram o método Pollutant Origin, Surcharge Hydraulically (POSH). Esse método classifica as fontes em três níveis de geração de carga contaminante: reduzida, moderada e elevada; considerando o tipo de atividade, sua capacidade geradora de contaminante e a carga hidráulica associada. Entretanto, a tentativa de controlar todas as atividades poluidoras em função de importantes propriedades físicoquímicas de contaminante e, sobretudo, para tornar heurístico o modus operandi da gestão da periculosidade de fontes contaminantes, alguns trabalhos propuseram a veiculação de índices (e.g. Zwahlen 2004; Andreo et al. 2006; Nobre et al. 2007; Mimi et al. 2009; Wang et al. 2012).
Portanto, os trabalhos revisados para classificar o grau de perigo direcionaram suas proposições com base numa relação de critérios entre a importância das propriedades físicoquímicas de fontes contaminantes. O produto dessa relação originou uma padronização do perigo que cada fonte contaminante pode representar em superfície.

Em síntese, essas questões apresentaram-se como um problema multicriterial, cuja finalidade foi classificar padrões de perigo. Contudo, algumas limitações podem ser traduzidas pela dificuldade operacional de inserir variados critérios (ou propriedade de contaminantes) e, ainda, na ponderação dos mesmos.

Assim sendo, propõem-se um modelo multi-criterial de reconhecimento de padrões, pelo príncípio de lógica fuzzy, referido em Zadeh (1967), com a inclusão de uma análise hierárquica de processo (AHP). Para tanto definiu-se uma área de aplicação para aferir a proposta de um modelo de risco: a cidade de Belém, Pará, Brasil.

\section{Características da Área de Estudo}

A proposta metodológica para avaliar e mapear o risco de contaminação das águas subterrâneas será aplicada na cidade de Belém e ilhas de Outeiro e Mosqueiro, que integram a região metropolitana de Belém (RMB). Essa região, pertence ao estado Pará, norte do Brasil; limitada ao sul e a oeste, pelo rio Guamá e baia de Guajará; cuja zona urbana foi delimitado em $1.64 \mathrm{~km}^{2}$, se situam 18 bacias hidrográficas. Os 
detalhes de características geológicas e hidrogeológicas podem ser consultadas em Matta (2002); Palheta (2008); Lisbôa et al., (2016).

Por outro lado, dados catalogados pelo Sistema de Informação de Águas Subterrâneas (SIAGAS), operado pela Companhia de Pesquisa e Recursos Minerais (CPRM) Serviço Geológico do Brasil, associado a 756 poços irregularmente distribuídos no referido domínio espacial, possibilitou a obtenção de duas importantes informações: (i) ocorrência da litologia da zona vadosa, a partir dos perfis litoconstrutivos de poços; e, (ii) os respectivos níveis hidroestáticos (Figuras 1A,B).

Figura 1 - (A) Litologia da zona vadosa; e, (B) Nível hidroestático de poços.

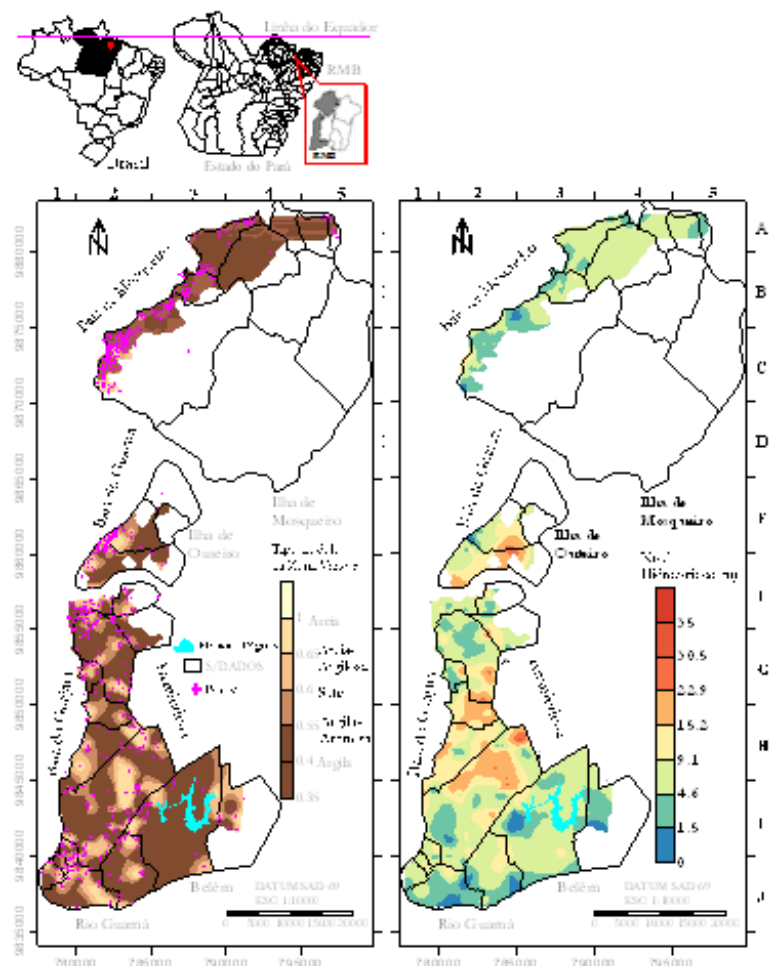

Fonte: Elaborado pelos Autores (2016).

Portanto, considerando a avaliação da vulnerabilidade intrínseca em aquíferos freáticos, pelas informações catalogadas, podese obter o índice $\mathbf{Y}$ através do método GOD (FOSTER, HIRATA, 1988).

Por essas características litológicas e hidroestáticas que a vulnerabilidade de aquíferos freáticos é solicitado a uma forte pressão ao ambiente subterrâneo, advindo da zona urbana e perirubana de Belém e ilhas de Outeiro e Mosqueiro. Essa pressão é associada a deficiência de ações estruturais e estruturantes no âmbito do esgotamento sanitário.

Atualmente, a zona urbana e periurbana da cidade apresenta valores inexpressivos do índice de atendimento com rede coletora e tratamento dos esgotos gerados. Segundo dados do Sistema Nacional de Informação do Saneamento (SNIS, 2012) revelaram que o município de Belém, de 1.432.844,0 habitantes (IBGE, 2014); dispõe de 8\% dos domicílios conectados a rede coletora e, do volume diário de esgoto gerado $\left(278.680 \mathrm{~m}^{3} / \mathrm{dia}\right)$, apenas $1,6 \%$ são tratados.

Pelos dados tabulados a partir de Dias (2007), associado a densidade populacional (habitantes $/ \mathrm{m}^{2}$ ), atualizado pelo dados de IBGE (2014); e, considerando a capacidade média de geração de $3 \mathrm{~kg} \mathrm{~N}-\mathrm{NO}_{3} /$ habitante (FOSTER, HIRATA, 1993), caracteriza-se a distribuição da capacidade geradora em $\mathrm{kg} \quad \mathrm{N}-\mathrm{NO}_{3} / \mathrm{m}^{2}$ (Figura 2A).

Em contraste com a abrangência da rede coletora de esgoto, cuja distribuição está ilustrada pela Figura 2B, tabulados a partir dos dados de IBGE (2000), nota-se uma possível situação de risco de contaminação das águas 
subterrâneas, função do baixo percentual de cobertura da rede de esgoto, em áreas com capacidade de geração de $\mathrm{N}-\mathrm{NO}_{3}$ superior a $0,07 \mathrm{~kg} / \mathrm{m}^{2}$.

Figura 2 - (A) Potencial gerador de N-NO3 $\left(\mathrm{kg} / \mathrm{m}^{2}\right)$; e, (B) Cobertura da rede de esgoto (\%).
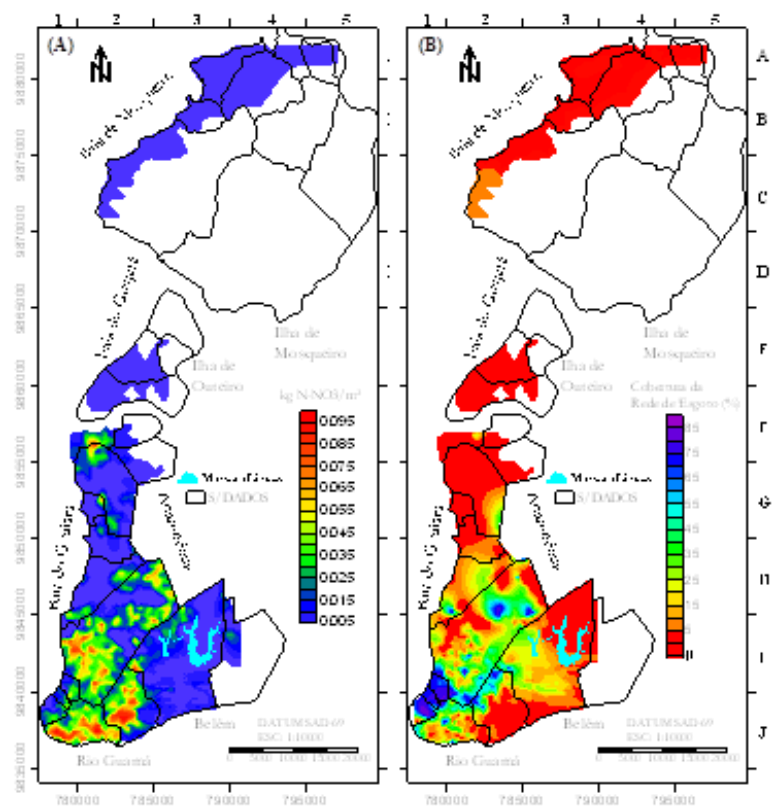

Fonte: Adaptado pelos Autores a partir de Dias (2007) e IBGE (2000)

No que se refere a cobertura da rede de esgoto sanitário existentes, o sistema é operado pela Companhia de Saneamento do Pará (COSANPA). Na área que corresponde a zona urbana de Belém, áreas de maior abrangência da rede coletora ainda referem-se a projetos, idealizados e parcialmente executados desde o "ciclo econômico da borracha" (final do século XIX e início do século XX), até Programas de Saneamento para Populações de Baixa Renda (PROSANEAR) e Ação Social em Saneamento (PROSEGE), financiados pela banca brasileira e interamericana, e pelo erário público (SILVA, 2005). Por outro lado, nas zonas insulares, além do abastecimento público de água ser realizado por captação subterrânea, duas estações de tratamento compõem o sistema de esgoto na ilha de Outeiro: uma atendendo uma demanda mais de 4.500 habitantes, contando com uma rede coletora de $3,5 \mathrm{~km}$; e, a outra de 2 km. Segundo a Agência de Regulação Municipal de Água e Esgoto (AMAE, 2014), apenas em uma parte da ilha de Mosqueiro há sistema de esgoto, que é composto por duas lagoas aeradas facultativas. Atualmente, aeradores não existem, o tanque de contato onde eram utilizados carvões ativados está deteriorado. Assim, de forma precária, funciona uma estação de tratamento com metade da capacidade de tratamento.

Nesses termos, aliada a questões potenciais de contaminação, no domínio espacial urbano e periurbano de Belém e ilhas, o aumento do número de poços para poder atender uma crescente demanda de abastecimento de água para múltiplos usos, pode ocasionar graves consequências ambientais e socioeconômicas. Logo, mapear o risco, como ação preventiva com fulcro a proteção dos recursos hídricos subterrâneos, apresenta-se como uma ferramenta de gestão ambiental.

\section{Avaliação da Vulnerabilidade Intrínseca}

Para avaliar o grau de vulnerabilidade intrínseca na zona urbana e periurbana da cidade de Belém e ilhas, recorre-se ao método GOD (Foster, Hirata, 1988). Considera-se o parâmetro $\mathrm{x}_{\mathrm{G}}$ igual a 1, i.e. para aquífero livre; e, o parâmetro $x_{O}$, referente ao tipo de solo da 
zona vadosa advindo dos perfis litoconstrutivo de poços; e de $\mathrm{x}_{\mathrm{D}}$, obtido pelo nível hidroestático de poços. A multiplicação entre $\mathrm{x}_{\mathrm{G}}, \mathrm{x}_{\mathrm{O}}$ e $\mathrm{x}_{\mathrm{D}}$ produz um índice $\mathbf{Y}$ sendo: $0 \leq \mathbf{Y} \leq$ 0,1 (insignificante "I"); $0,1 \leq \mathbf{Y} \leq 0,3$ (baixo "B"); $0,3 \leq \mathbf{Y} \leq 0,5$ (média "M"); $0,5 \leq \mathbf{Y} \leq 0,7$ (alta “A”); e, $\mathbf{Y} \geq 0,7$ (extremo "E”). Interpolase o índice $\mathbf{Y}$ pelo aplicativo via método kriging, obtendo-se o mapa de vulnerabilidade intrínseca. Para quantificar o grau de perigo propõem-se um modelo multi-criterial fuzzyAHP. Para tanto, define-se uma matriz padrão de perigo.

\section{Definição de um Padrão de Perigo}

Enquadra-se o potencial gerador de N$\mathrm{NO}_{3}$ num padrão de perigo de contaminação das águas subterrâneas, definido em cinco classes (muito baixo "MB", baixo "B”, médio "M", alto "A", e muito alto "MA") e função de cinco critérios que são associados às seguintes propriedades físico-químicas:

Toxicidade (p1): expressa em $\mu \mathrm{g} / \mathrm{L}$, refere-se aos efeitos adversos provocados pela interação de químicos com organismo humano. Assim, quanto menor a concentração permitida na água potável, mais tóxica é ação de um dado composto;

- Mobilidade (p2): utiliza-se a concepção do coeficiente de partição água-octanol (KOC), associado à mobilidade de um dado composto e seu deslocamento em direção ao aquífero. Portanto, quanto menor for o valor de KOC, numa escala logarítmica, o composto tende a ser mais recalcitrante, i.e. negligenciável adsorção por solos e sedimentos, e potencial de rápida migração para águas subterrâneas;

- Degradabilidade (p3): refere-se às degradações químicas (por hidrólise e fotoxidação) e bioquímicas (por biodegradação, aeróbica ou anaeróbica). A quantificação desse parâmetro pode ser calculada por meio do aplicativo EPI Suite ${ }^{\mathrm{TM}}$ v4.11, que produz um índice de degradabilidade, desenvolvido pela US. EPA (US. EPA, 2011). Portanto, quanto menor for o índice, maior será a resistência do composto às degradações químicas e/ou bioquímicas;

- Solubilidade (p4) define-se pela concentração saturada de um composto na água, condicionada a uma temperatura e pressão, que é inversamente proporcional a KOC. Assim sendo, essa propriedade indica que compostos com elevada solubilidade são mais suscetíveis à biodegradação e sorção de solos e sedimentos;

- Volume/quantidade de N-NO3 (p5): esta variável parte do conhecimento de que em média uma pessoa produz $3 \mathrm{~kg}$ de N-NO3/ano (FOSTER, HIRATA, 1993). Assim, pode-se estimar a quantidade anual de produção desse composto por área, função da densidade populacional (habitante/m²).

\section{Modelo Multi-Criterial Fuzzy-AHP}

Sendo n o número de propriedade físicoquímica de contaminantes $(\mathrm{p})(\mathrm{p}=1,2, \ldots, \mathrm{n})$, e $\mathrm{m}$ o número de classes $(\mathrm{h}=1,2, . ., \mathrm{m})$ convenientes para classificar o perigo; o termo $\mathrm{zp}, \mathrm{h}$ representa o valor de cada $\mathrm{p}$ enquadrado 
numa classe $h$, de tal modo que se constitua numa matriz $\mathrm{Zp}$, denominada como matriz padrão. Para construir Zp, adotou-se o padrão referido por Wang et al. (2012) para as quatros primeiras propriedades.

$$
Z_{p}=\left[\begin{array}{ccccc}
1,1.10^{6} & 10^{6} & 10^{3} & 10^{2} & 10 \\
5 & 4,5 & 3,5 & 2,5 & 1,5 \\
5 & 4,25 & 3,25 & 2,25 & 1,75 \\
10^{-3} & 10^{-1} & 10 & 10^{4} & 10^{6} \\
0,010 & 0,015 & 0,030 & 0,060 & 0,090
\end{array}\right]
$$

Entretanto, aos termos $\mathrm{Z}_{\mathrm{p}, \mathrm{h}}$ da matriz $\mathrm{Z}_{\mathrm{p}}$ faz-se necessário uniformizá-los, quer por um processo de normalização ou por harmonização. Portanto, quando graus de perigo aumentam com o incremento das propriedades, procede-se a normalização; e, quando diminuem, faz-se a harmonização, respectivamente, por:

$$
z_{p, h^{\prime}}=\frac{z_{p, h}-z_{p, 1}}{z_{p, 5}-z_{p, 1}} \quad z_{p, h^{\prime}}=\frac{z_{p, 5}-z_{p, h}}{z_{p, 5}-z_{p, 1}}
$$

Em que $\mathrm{z}_{\mathrm{p}, \mathrm{h}}$ ' está associado à matriz de perigo; o qual pode ser normalizado e/ou harmonizado. Se $\mathrm{z}_{\mathrm{p}, \mathrm{h}}$ ' $=1$, uma propriedade pertence totalmente a uma classe $\mathrm{h}$, enquanto que, se $\mathrm{z}_{\mathrm{p}, \mathrm{h}}{ }^{\prime}=0$, uma propriedade não pertence totalmente a classe $h$. Os termos $z_{p, 5}$ e $z_{p, 1}$ referem-se aos termos de maior e menor periculosidade, respectivamente. Portanto, transforma-se a matriz $\mathrm{Z}_{\mathrm{p}}$ em $\mathrm{Z}_{\mathrm{p}}$.

$$
Z_{p}{ }^{\prime}=\left[\begin{array}{ccccc}
1 & 0,91 & 10^{-3} & 10^{-4} & 0 \\
1 & 0,86 & 0,57 & 0,29 & 0 \\
1 & 0,77 & 0,46 & 0,15 & 0 \\
1 & 1 & 1 & 0,99 & 0 \\
1 & 0,94 & 0,75 & 0,38 & 0
\end{array}\right]
$$

Assim sendo, o modelo utilizado para efetuar o reconhecimento (ou enquadramento) aos padrões de perigo, baseia-se na proposta de Chen (1996). Portanto, inicialmente, assume-se uma matriz de dados $X_{P}$, cujos termos $x_{p, j}$ referem-se à matriz de dados do perigo. Os dados referem-se ao grid regular advindo do mapa do potencial gerador expresso em $\mathrm{kg} \mathrm{N}$ $\mathrm{NO} 3 / \mathrm{m}^{2}$. Em seguida efetua-se a uniformização dos termos $\mathrm{x}_{\mathrm{p}, \mathrm{j}}$ da matriz $\mathrm{X}_{\mathrm{P}}$.

$$
x_{p, j}{ }^{\prime}=\frac{x_{p, j}-z_{p, 1}}{z_{p, 5}-z_{p, 1}} \quad x_{p, j}{ }^{\prime}=\frac{z_{p, 5}-x_{p, j}}{z_{p, 5}-z_{p, 1}}
$$

Assim, obtem-se uma matriz $\mathrm{X}_{\mathrm{P}}$, cujo termo seja $x_{p, j}$, de tal modo que pode ser considerada como uma matriz do grau de pertinência das propriedades p. Portanto, assumindo que o grau de pertinência de cada amostra $\mathrm{j}$ deve pertencer a uma dada classe $\mathrm{h}$ de perigo, considera-se uma matriz $U$, cujos termos designam-se por $\mathrm{u}_{\mathrm{h}, \mathrm{j}}$. Para classificar o perigo, o valor de h é igual a 5, uma vez que se considera a existência de cinco classes: "MB" (1), "B" (2), “M" (3), “A” (4) e "MA" (5). Assim, Chen (1996) considerou que, de acordo com a teoria dos conjuntos fuzzy (ZADEH, 1967), a matriz U fica sujeita a seguinte restrição:

$$
\sum_{h=1}^{5} u_{h, j}=1
$$

Por essa restrição, cada propriedade $\mathrm{p}$ têm uma dada importância na classificação do perigo. Nesses termos, o modelo de reconhecimento de padrão, com um princípio 
fuzzy, proposto por Chen (1996) considera que existe uma distância que separa uma amostra $\mathrm{j}$ do seu grau h, igual a:

$$
d_{h, j}=\left\{\sum_{p=1}^{p}\left[w_{p} \cdot\left(x_{p, j}{ }^{\prime}-z_{p, h}{ }^{\prime}\right)\right]^{2}\right\}
$$

Em que $w_{p}$ refere-se ao peso que cada propriedade. A atribuição dos pesos foi aferida por análise hierárquica de processo (AHP), pelo julgamento de importância de cada propriedade p na composição do perigo, conforme propôs Saaty (2008).

Desse modo, avalia-se qual das propriedades físico-químicas apresenta maior peso na composição final na quantificação do perigo de contaminação das águas subterrâneas. Considerando o potencial gerador de $\mathrm{N}-\mathrm{NO}_{3}$ a solubilidade é uma propriedade mais importante que as demais. Assim, obteve-se $\mathrm{w}_{\mathrm{p} 1}=0,03$, $\mathrm{w}_{\mathrm{p} 2}=0,13, \mathrm{w}_{\mathrm{p} 3}=0,08, \mathrm{w}_{\mathrm{p} 4}=0,51$ e $\mathrm{w}_{\mathrm{p} 5}=0,25$.

Definido os pesos das propriedades físico-químicas $\left(\mathrm{w}_{\mathrm{p}}\right)$; considera-se que, do ponto de vista da teoria fuzzy, o termo $\mathrm{u}_{\mathrm{h}, \mathrm{j}}$ como um peso para $\mathrm{d}_{\mathrm{h}, \mathrm{j}}$. Assim, define-se uma distância ponderada $\left(D_{h, j}\right)$ que separa uma amostra $\mathrm{j}$ da sua classe $\mathrm{h}$, igual a $\mathrm{u}_{\mathrm{h}, \mathrm{j}}$. $\mathrm{d}_{\mathrm{h}, \mathrm{j}}$. Para resolver $\mathrm{u}_{\mathrm{h}, \mathrm{j}}$, estabelece o mínimo de uma função objetivo expressa por:

$$
\min \left\{F\left(u_{h, j}\right)=\sum_{h=1}^{5} D_{h, j}^{2}\right\}
$$

Como referiu Chen (1996), de modo a obter um lagrangiano para otimizar o processo de classificação do perigo, por restrição, temse:

$$
L\left(u_{h, j}, \lambda_{j}\right)=\sum_{h=1}^{5} D_{h, j}^{2}-\lambda_{j}\left(\sum_{h=1}^{5} u_{h, j}-1\right)
$$

Em que $\lambda_{j}$ é um multiplicador de lagrange. Assim, a resolução do valor ótimo que enquadra uma amostra $\mathrm{j}$ a um grau de pertinência $h$, por meio de $u_{h, j}$, atende-se por duas condições: (i) $\partial \mathrm{L}\left(\mathrm{u}_{\mathrm{h}, \mathrm{j}}, \lambda_{\mathrm{j}}\right) / \partial \mathrm{L}\left(\mathrm{u}_{\mathrm{h}, \mathrm{j}}\right)=0$; (ii) $\mathrm{L}\left(\mathrm{u}_{\mathrm{h}, \mathrm{j}}, \lambda_{\mathrm{j}}\right) / \partial \mathrm{L}\left(\lambda_{\mathrm{j}}\right)=0$. Pelas derivadas parciais da função de lagrange, obtem-se a função de pertinência de $u_{h, j}$ para uma classe $h$, por:

$$
u_{h, j}=\left(d_{h, j}^{2} \sum_{h=1}^{5} \frac{1}{d_{h, j}^{2}}\right)^{-1}
$$

Sendo que esse equação está condicionada $a d_{h, j} \neq 0$. E, quando $d_{h, j}=0$, significa que a amostra j pertence a um nível $\mathrm{h}$, então $u_{h, j}$ é igual a 1. Entretanto, pela equação acima, pode-se efetuar a classificação apenas pelos extremos das classes h (i.e. uma matriz de amostras $X_{P}$ poderia ser enquadrada em dois níveis de classes $\mathrm{h}$, correspondente a matriz padrão $\mathrm{Z}_{\mathrm{P}}$, ou seja, $\mathrm{u}_{\mathrm{h}, \mathrm{j}}=0$ ou $\mathrm{u}_{\mathrm{h}, \mathrm{j}}=1$ ).

Para que a matriz de dados possam ser enquadradas num intervalo entre 0 e 1 , de tal modo que possam corresponder às categorias de perigo nas variadas classes $h$, e que sejam associadas aos respectivos valores característicos (categoria 1, 2, 3, 4 a 5), considera-se: (i) $\mathrm{u}_{\mathrm{h}, \mathrm{j}}$ * como termos da matriz U*, que representa uma matriz de pertinência de cada amostra $\mathrm{j}$ que pode pertencer a cada 
classe h, variando de 0 a 1; (ii) a classificação numa das categorias $\mathrm{h}$ não pode ser efetuado diretamente pela matriz, o valor dos termos $\mathrm{u}_{\mathrm{j}}{ }^{*}$ é igualado a $\left(\mathrm{u}_{1, \mathrm{j}}, \mathrm{u}_{2, \mathrm{j}}, \mathrm{u}_{3, \mathrm{j}}, \mathrm{u}_{4, \mathrm{j}}, \mathrm{u}_{5, \mathrm{j}}\right)^{\mathrm{T}}$. Assim, o referido vetor $\mathrm{u}_{\mathrm{j}}^{*}$ associado as categorias $h$, obtem-se um índice de perigo - $\mathrm{I}_{\mathrm{j}(\mathrm{P})}$, igual a:

$$
I_{j(P)}=(1,2,3,4,5) \cdot u_{j}^{*}=\sum_{h=1}^{5} u_{h, j}^{*} \cdot h
$$

Sendo $\mathrm{I}_{\mathrm{j}(\mathrm{P})}$ igual a 1 "MB", 2 "B", 3 "M", 4 "A" e 5 "MA". Um das vantagens de aplicar o modelo de reconhecimento de padrão fuzzy-AHP deve-se ao fato de que, o perigo é função de uma condição ponderada entre propriedades físico-químicas.

Por outro lado, a veiculação de um índice propicia a sua interpolação num determinado domínio espacial, pelo grid regular advindo do mapa do potencial gerador expresso em kg N-NO $3 / \mathrm{m}^{2}$.

A respeito das propriedades do composto $\mathrm{N}-\mathrm{NO}_{3}$, Sara (1994) referiu que o valor de $p_{1}$ é igual a $2.10^{4} \mu \mathrm{g} / \mathrm{L}$, enquanto que $\mathrm{p}_{2}$ e $\mathrm{p}_{3}$ foi sugerido valores iguais a 1,50 e 2,75 , respectivamente. Para $\mathrm{p}_{4}, \mathrm{o}$ referido composto apresenta altos valores, sendo adotado um valor igual a $10^{6} \mu$ moles/L.

\section{Análise do Risco de Contaminação}

A avaliação do risco de contaminação das águas subterrâneas define-se como sendo igual à relação entre graus de vulnerabilidade intrínseca e de periculosidade (Quadro 1).
Quadro 1 - Matriz do Risco Básico de Contaminação das Águas Subterrâneas.

\begin{tabular}{|c|c|c|c|c|c|}
\hline \multirow{2}{*}{ Perigo $-\mathbf{I}_{\mathbf{j}(\mathbf{P})}$} & \multicolumn{5}{|c|}{ Vulnerabilidade $-\mathbf{Y}_{\mathbf{G O D}}$} \\
\cline { 2 - 6 } & $\mathbf{I}$ & $\mathbf{B}$ & $\mathbf{M}$ & $\mathbf{A}$ & $\mathbf{E}$ \\
\hline MA & $\mathrm{M}$ & $\mathrm{M}$ & $\mathrm{A}$ & $\mathrm{MA}$ & $\mathrm{MA}$ \\
\hline A & $\mathrm{B}$ & $\mathrm{M}$ & $\mathrm{A}$ & $\mathrm{MA}$ & $\mathrm{MA}$ \\
\hline M & $\mathrm{MB}$ & $\mathrm{B}$ & $\mathrm{M}$ & $\mathrm{A}$ & $\mathrm{A}$ \\
\hline B & $\mathrm{MB}$ & $\mathrm{B}$ & $\mathrm{B}$ & $\mathrm{M}$ & $\mathrm{A}$ \\
\hline MB & $\mathrm{MB}$ & $\mathrm{MB}$ & $\mathrm{B}$ & $\mathrm{B}$ & $\mathrm{M}$ \\
\hline
\end{tabular}

O grau de alta prioridade para ampliação da rede de esgotamento sanitário é requerido caso o risco de contaminação seja classificado como "MA" e "A". A prioridade de ampliação da rede pode ser associada a uma cobertura superior a $70 \%$ da área de risco.

Caso áreas classificadas com os respectivos graus de risco já sejam abrangidas com o referido percentual de cobertura, recomenda-se que seja implementado um plano de gestão que incorpore um percentual superior a $50 \%$ das condutas do sistema. Refere-se que esse plano deva ter como principal objetivo evitar vazamentos de esgoto não tratado, através de inspeções de eventuais falhas estruturais das condutas, de conexões indevidas de condutas de águas pluviais e/ou águas residuais domésticas e industriais (afluências indevidas).

Quando o risco de contaminação das águas subterrâneas for classificado como "M", “B” ou "MB", a prioridade de ampliação à rede de esgoto pode ser classificada em média até baixa. A prioridade de ampliação da rede de esgoto pode ser associada a uma cobertura igual ou inferior a $70 \%$ da área de risco Entretanto, caso as áreas classificadas com os respectivos 
graus de risco já sejam abrangidas com o referido percentual de cobertura da rede de esgoto, recomenda-se que seja implementado um plano de gestão que incorpore um percentual igual ou inferior a $50 \%$ das condutas do sistema.

\section{Resultados e Discussão}

O mapa da vulnerabilidade intrínseca de aquíferos revelou que, pouco mais de $70 \%$ da zona urbana e periurbana da cidade de Belém é vulnerável a contaminantes, mas somente quando continuamente lançados ou lixiviados, conforme a classificação moderada é referenciada por Foster e Hirata (1988).

Menos de $30 \%$ da zona urbana mapeada apresentaram-se como de alta vulnerabilidade intrínseca a contaminação de aquíferos freático. $\mathrm{Na}$ ilha de Outeiro e Mosqueiro, mais de $70 \%$ e 90\% da área foram classificadas como de vulnerabilidade grau "M", respectivamente (Figura 3A).

O grau alto de perigo, associado ao potencial gerador de $\mathrm{N}-\mathrm{NO}_{3}$, na zona urbana e periurbana da cidade de Belém, abrange uma área próximo de $40 \%$, face aos mais de $50 \%$ classificada como sendo de moderada periculosidade à contaminação. Refere-se que, em pelo menos quatro bacias hidrográficas, algumas zonas com alta densidade populacional foram classificadas como sendo de perigo "MA" (Figura 3B).

Entretanto, quando associado a vulnerabilidade intrínseca ao perigo, quase $40 \%$ da zona urbana indicaram ser áreas de alto risco de contaminação das águas subterrâneas. Nesses termos, zonas urbanas e periurbanas das ilhas de Outeiro e Mosqueiro que foram classificadas como de moderada periculosidade; quando associado a alta vulnerabilidade, algumas áreas indicaram alto risco de contaminação (Figura 4).

Figura 3 - A) Vulnerabilidade Intrínseca; e, B) Perigo de Contaminação pelo potencial gerador de $\mathrm{N}-\mathrm{NO}_{3}$.

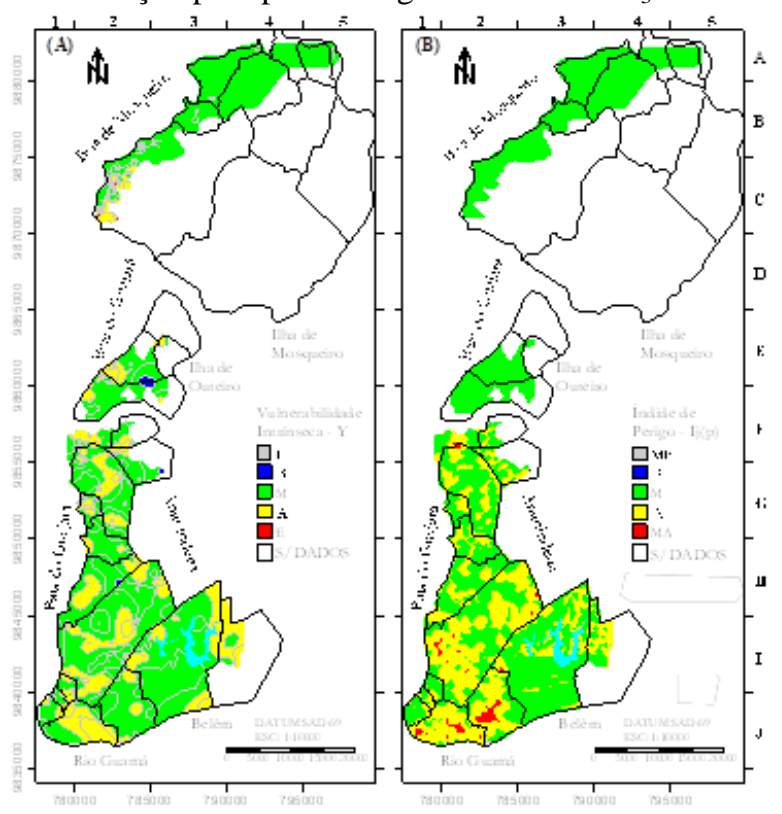

Fonte: Elaborado pelos Autores (2016).

Figura 4 - Mapa do Risco de Contaminação.
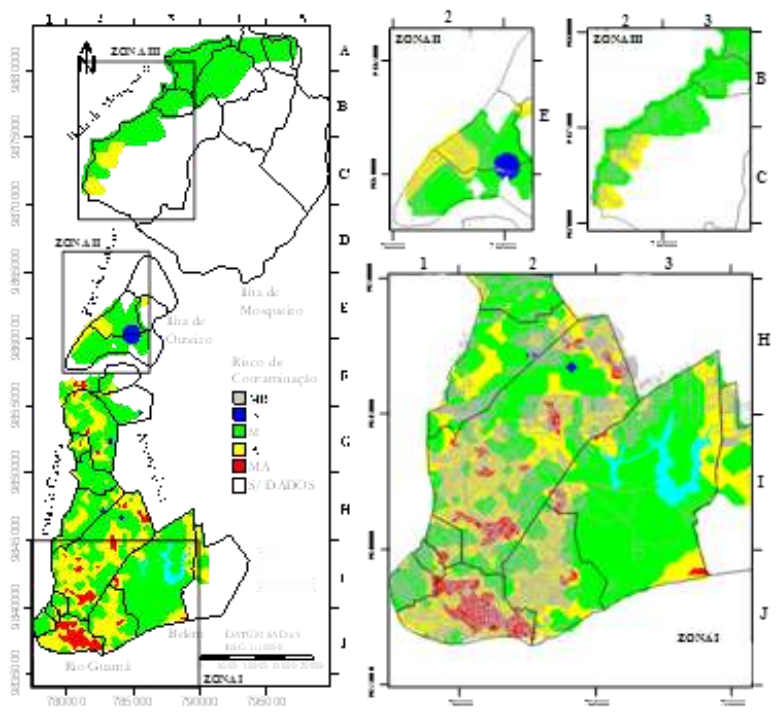

Fonte: Elaborado pelos Autores (2016). 
Os percentuais de abrangência do grau de vulnerabilidade intrínseca, perigo e do risco da zona urbana/periurbana de Belém e ilhas pode ser consultado nas Quadro 2, 3 e 4.

Enfatizaram-se três zonas. A primeira zona (ZONA I) ampliou-se a visualização do mapa de risco de uma área mais densamente urbanizada da cidade de Belém, de tal modo que observou-se áreas de risco muito alto "MA" e alto "A" de contaminação das águas subterrâneas.

Quadro 2 - Abrangência (\%) do Grau de Vulnerabilidade Intrínseca.

\begin{tabular}{|c|c|c|c|c|c|}
\hline $\begin{array}{c}\text { Zona } \\
\text { Urbana }\end{array}$ & I & B & M & A & E \\
\hline Belém & 0,0 & 0,62 & 70,05 & 29,33 & 0,0 \\
\hline Outeiro & 0,0 & 3,77 & 74,49 & 21,74 & 0,0 \\
\hline Mosqueiro & 0,0 & 0,0 & 92,0 & 7,23 & 0,77 \\
\hline
\end{tabular}

Quadro 3 - Abrangência (\%) do Grau de Periculosidade à Contaminação.

\begin{tabular}{|c|c|c|c|c|c|}
\hline $\begin{array}{c}\text { Zona } \\
\text { Urbana }\end{array}$ & MB & B & M & A & MA \\
\hline Belém & 0,0 & 0,0 & 57,26 & 39,41 & 3,33 \\
\hline Outeiro & 0,0 & 0,0 & 100 & 0,0 & 0,0 \\
\hline Mosqueiro & 0,0 & 0,0 & 100 & 0,0 & 0,0 \\
\hline
\end{tabular}

Quadro 4 - Abrangência (\%) do Grau de Risco de Contaminação das Águas Subterrâneas.

\begin{tabular}{|c|c|c|c|c|c|}
\hline $\begin{array}{c}\text { Zona } \\
\text { Urbana }\end{array}$ & MB & B & M & A & MA \\
\hline Belém & 0,0 & 0,30 & 49,70 & 39,90 & 10,10 \\
\hline Outeiro & 0,0 & 5,90 & 65,50 & 28,60 & 0,0 \\
\hline Mosqueiro & 0,0 & 0,0 & 93,40 & 6,60 & 0,0 \\
\hline
\end{tabular}

Entretanto, nessas área, a cobertura da rede de esgoto é inferior a 60\%, como visualiza-se pelo mapa ilustrado pela Figura $2 \mathrm{~B}$. Portanto, pelo modelo de análise de risco proposto, deve-se priorizar a ampliação da rede de esgoto para uma cobertura superior a $70 \%$.
Por outro lado, zonas classificadas como sendo de grau "M" de risco de contaminação, a cobertura da rede de esgoto é igual ou superior a $70 \%$, de tal modo que se recomenda que seja implementado um plano de gestão que incorpore um percentual igual ou inferior a $50 \%$ das condutas do sistema existente.

O mapeamento que enfatizou a ZONA II e III revelou que, embora grande parte da área tenha sido classificada como sendo de risco moderado, em áreas de alto risco a cobertura de rede de esgoto é inferior a $5 \%$. Assim sendo, nessas áreas a prioridade de ampliação da rede pode ser associada a uma cobertura superior a $70 \%$, ou até igual ou inferior a 70\%, em áreas classificadas como sendo de grau " $M$ " ou "B" risco de contaminação.

A necessidade de validação do modelo de risco é geralmente recomendado pela World Health Organization (WHO, 1993), e pode ser efetuado pela associação entre resultados dos índices produzidos por sistema de indexação para avaliar a vulnerabilidade intrínseca, e resultados analíticos padrões de amostras de águas subterrâneas de poços.

Contudo, alguns trabalhos encontraram uma limitada correlação entre parâmetros hidrogeológicos que compõem sistemas de indexação; e, dados de qualidade das águas subterrênas (e.g. HOLDEN et al., 1992); bem como, resultados satisfatórios também foram obtidos (e.g. LIN et al., 1999). Entretanto, mesmo após processo de validação, um modelo de risco pode ainda apresentar-se subjetivo. 
Por essas perspectivas, o modelo proposto para avaliar o risco de contaminação das águas subterrâneas foi condicionado a um conceito pronunciado por Konikow e Bredehoeft (1992); de tal modo que o referido modelo é sujeito a ser melhorado por um processo de invalidação.

Portanto, o processo de validação e verificação deve ser substituído pelo termo history maching. Esse termo abrange o processo de calibração e validação sem a conotação da exatidão e verossimilhança. Logo, o modelo proposto neste trabalho apresentou resultados temporários, pois foram baseados num potencial gerado de $\mathrm{N}_{-} \mathrm{NO}_{3}$, função da densidade populacional que pode expandir-se ou retrair-se. Ainda assim, as ações a serem deliberadas pela classificação do risco, nomeadamente a priorização por ampliar a rede de esgoto e incorporação de um plano de gestão na rede existente, podem sofrer atualizações.

Nesse âmbito, Saidi et al. (2011) referiram que o perigo tem um caráter "dinâmico", enquanto que a vulnerabilidade intrínseca tem um caráter "estático". O dinamismo do perigo, relacionado ao potencial gerador de nitrogênio-nitrato, está associado ao aumento ou diminuição da densidade populacional. Assim sendo, quando inserido numa determinada área com um dado grau de vulnerabilidade produz-se o risco, condicionado pela classificação do perigo.

\section{Conclusões e Recomendações}

Esse trabalho desenvolveu uma metodologia para mapear o risco de contaminação das águas subterrâneas, a partir da associação entre a vulnerabilidade intrínseca e perigo do potencial gerador de nitrogênionitrato em zonas urbanas e periurbanas. A vulnerrabilidade intrínseca foi avaliada pelo método GOD, e o para classificar o grau de periculosidade recorreu-se a uma proposta de um modelo multi-critério de reconhecimento de padrões, com um princípio fuzzy, no qual inseriu-se um análise AHP. Em ambos, gerouse um índice, que por meio de métodos geoestatísticos, sendo produzido o mapa de vulnerabilidade e do perigo. Em seguida, a associação de ambos os mapas, produziu-se o mapa de risco de contaminação das águas subterrâneas.

A metodologia aplicada é genérica a ponto de ser aplicada, novamente, em qualquer domínio espacial e hidrogeológico. Entretanto, ressalta-se que outros métodos de indexação para avaliar a vulnerabilidade intrínseca pode ser utilizado, desde que haja disponibilidade de dados hidrológicos e hidrogeológicos. Outras propriedades físico-química do contaminante, nomedamente o nitrogênio-nitrato, podem ser inseridos no modelo fuzzy-AHP, e.g. tempo de meia-vida.

Por outro lado, o tipo de classificação do perigo refere-se a um conceito de perigo aparente, pois se pode considerar que numa determinada fonte, o contaminante possa ficar em estado "latente". 
Assim sendo, pode ser que o mesmo dificilmente entre em contato com ambiente subterrâneo, caso sejam adotado critérios ou dispositivos de segurança que possam evitar e/ou monitorar vazamentos (e.g. abrangência de rede coletora de esgotamento sanitário, a inclusão na rede existente de um plano de gestão de condutas, bem como, o potencial de infiltração de esgoto ou água residual, que pode ser expresso pela relação entre vazão de pico e escoamento superficial numa bacia hidrográfica).

Logo, recomenda-se a inclusão do conceito de suscetibilidade. Assim, além de produzir um conceito de risco real, quando associado ao grau de reserva (ou disponibilidade) e qualidade da água subterrânea, pode-ser originar um risco efetivo de contaminação.

Portanto, de um modo geral, a proposta apresentam-se como uma ferramenta para ampliar a consciência dos usuários do sistema sobre a vulnerabilidade de aquíferos, perigo e risco de contaminação; e, o entendimento do público sobre a necessidade de proteger a água subterrânea para evitar impactos ambientais e socioeconómicos. Assim sendo, a proposta metodológica do modelo de análise do risco de contaminação permitiu estabelecer um elo entre os usuários do sistema e tomadores de decisão pela síntese e simplificação das condições hidrogeológicas, periculosidade e risco de contaminação, via mapas temáticos enfatizam a distribuição espacial dessas informações.

\section{Referências Bibliográficas}

ALLER, L., BENNET, T., LEHR, H. J., PETTY, J. R., and HACKETT, G. (1987). "DRASTIC; A standardized system for evaluating groundwater pollution potential using hydrogeologic settings.'US.EPA-600/2$87-035,622 \mathrm{pp}$.

AMAE. Agência Reguladora Municipal de Água e Esgoto de Belém. 2014. Disponível em: $<$ http://amae.belem.pa.gov.br/?p=902>. Acesso em: jun de 2016 .

ANDREO, B; GOLDSCIDER, N; VADILLO, I ET AL., 2006. "Karst groundwater protection: First application of a Pan-European approach to vulnerability, hazard and risk mapping in the Sierra de Líbar (Southern Spain)". Science of the Total Environment, v. 357, pp. 54-73.

AUGE, M. Vulnerabilidad de acuíferos: conceptos y metodos. 2004. Buenos Aires: Universidade de Buenos Aires.

CHEN, S. Y. (1996) Non-structured decision making analysis and fuzzy optimum seeking theory for multi-objective systems. J. Fuzzy Math. 4(2), 835-842.

DIAS, Mario Benjamin. Urbanização e ambiente urbano no distrito administrativo de Icoaraci, Belém-PA. 2007. Tese (Doutorado em Geografia Humana) - Faculdade de Filosofia, Letras e Ciências Humanas, Universidade de São Paulo, São Paulo, 2007. doi:10.11606/T.8.2007.tde-03122007-11424.

DOERFLIGER, N.; ZWAHLEN, F. EPIK: a new method for outlining of protection areas in karstic environment. In: Günay, G.; Jonshon, A.I. (Ed). International symposium and field seminar on karst waters and environmental impacts. Antalya, Turkey, Balkema, Rotterdam, 1997, 117-123 p

FOSTER, S. S. D. e R. HIRATA. 1988.

Groundwater pollution risk assessment: a methodology using available data. WHOPAHO/ HPE-CEPIS Technical Manual. Lima, Peru. 
FOSTER, S. S. D e HIRATA, R. C. A.

Determinação do Risco de Contaminação das

Águas Subterrâneas: Um Método Baseado em

Dados Existentes. São Paulo - Instituto

Geológico, P. il., tabs. $23 \mathrm{~cm}$ (Boletim, 10),

1993.

FOSTER, S.; HIRATA, R.; GOMES, D.;

D'ELIA, M.; PARIS, M. Underground Water

Quality Protection: a Guide for Water Utilities,

Municipal Authorities and Environment

Agencies. Washington DC, USA: World Bank

Publication, 2002.

GODOY, M. C. T. F. Estudo Hidrogeológico

das Zonas Não Saturada e Saturada da

Formação Adamantina em Presidente Prudente,

Estado de São Paulo, São Paulo, Tese

(Doutorado em Recursos Minerais e

Hidrogeologia), Instituto de Geociências,

Universidade de São Paulo, 1999, 156 p.

HOLDEN, L.R; GRAHAM, J.A; WHITMORE, R.W; ALEXANDER, W.J; PRATT, R.W;

LIDDLE, S.K and PIPER, L.L. 1992. Results of the National Alachlor Well Water Survey.

Environmental Science \& Technology 26. 935-

943.

Instituto Brasileiro de Geografia e Estatística (IBGE), Pesquisa nacional de saneamento básico, 2000. Disponível em:

<http://www.ibge.gov.br/ibgeteen>. Acesso em: ago de 2016.

Instituto Brasileiro de Geografia e Estatística (IBGE). Censo Demográfico 2014. Disponível em:

<http://www.ibge.gov.br/home/estatistica/indic adores/pib/defaultcnt.shtm>. Acesso em: ago. 2016.

KONIKOW, L.F., and BREDEHOEFT, J.D., 1992, Ground-water models cannot be validated, Advances in Water Resources, 15, pp. 75-83.

LERNER,D. N.; YANG,Y .; BARRETT, M. H.; TELLAM, J. H. Loading of non-agricultural nitrogen in urban groundwater, IAHS-AISH Publication. 259; 117-123. 1999.
LIN, H.S; SCOTT, H.D; STELLE, K. F;

INYANG, H.I, (1999). Agricultural chemicals in the alluvial aquifer of a typical country of the Arkansas delta. Journal of Environmental Monitoring and Assessment, n. 58, pp. 151 172.

LISBOA, É.G; CARVALHO, J. M C. M; MENDES, R. L. R. O Uso da Geoestatística na Avaliação dos Parâmetros Hidrogeológicos para Compor o Mapa de Vulnerabilidade Intrínseca de Aquíferos. RBRH, Porto Alegre, v. 21, n. 1, p. 188-199, Mar. 2016.

MATTA, M. A. S. Fundamentos hidrogeológicos para a gestão integrada dos recursos hídricos da Região de Belém/Ananindeua - Pará, Brasil. 2002. 584 f. Tese (Doutorado em Hidrogeologia). Centro de Geociências, Universidade Federal do Pará, 2002.

MIMI, Z.A; ASSI, A. Intrinsic vulnerability, hazard and risk mapping for karst aquifers: a case study. Journal of Hydrology, 364 (2009), pp. $298-310$.

NOBRE. R.C.M; ROTUNNO, O.C; MANSUR, W.J; NOBRE, M.M.M; COSENZA, C.A.N. Groundwater vulnerability and risk mapping using GIS, modeling and a fuzzy logic tool. Journal of Contaminant Hydrology, 94 (2007), pp. 277-292.

PALHETA, E. S. M. Estudo da compartimentação e arcabouço neotectônico da ilha de Mosqueiro - Pará empregado no conhecimento hídrico subterrâneo. 2008. $241 \mathrm{f}$. Tese (Doutorado em Geologia e Geoquímica). Instituto de Geociências, Programa de PósGraduação em Geologia e Geoquímica, Universidade Federal do Pará, Belém. 2008.

SAATY, T.L. Decision making with the analytical hierarchy process International Journal of Services Sciences., 1 (1) (2008), pp. 83-98

SAIDI S, BOURI S, BEN DHIA H, ANSELME B. Assessment of groundwater risk using intrinsic vulnerability and hazard mapping: application to Souassi aquifer, 
Tunisian Sahel. Agric Water Manage 2011;98:1671-82.

SARA, M. N. (1994). Standard Handbook for Solid and Hazardous Waste Facility

Assessments. Lewis Publishers. CRC. Press.

SILVA, V. S. Plano Diretor Setorial do Sistema de Esgotamento Sanitário: Alternativas de Concepção para a Área de Maior Adensamento Populacional do Município de Belém-PA. 199

f. Dissertação (Mestrado em Engenharia Civil). Centro Tecnológico, Programa de PósGraduação em Engenharia Civil, Universidade Federal do Pará, Belém. 2005

SNIS. Diagnóstico de manejo de resíduos sólidos. Brasília: Sistema Nacional de Informação sobre Saneamento, 2012.

Disponível em: http://www.snis.gov.br/. Acesso em: jun de 2016.

U.S. EPA (United States Environmental Protection Agency). "Guide for conduction contaminant source inventories for public drinking water supplies: technical assistance document." Environmental Protection Agency, Office of Water, Washington, D.C, 1991.

U.S. EPA. Estimation Programs Interface Suite $^{\mathrm{TM}}$ for Microsoft ${ }^{\circledR}$ Windows, v 4.10; 2011. Washington, DC, USA

WANG, J; HE, J; CHEN, H. 2012. Assessment of groundwater contamination risk using hazard quantification, a modified DRASTIC model and groundwater value, Beijing Plain, China. Science of The Total Environment 188-189(14), pp. $74-95$.

WHITEHEAD, E.; HISCOCK, K.; DENNIS, P. Evidence for sewage contamination of the Sherwood Sandstone aquifer beneath Liverpool, UK, IAHS-AISH Publication. 259; Pages 179-185. 1999.

WHO (World Health Organization) 1993.

WHOQOL: study protocol. MNH/PSF/93.9. WHO, Genebra. 39 pp.

ZADEH, L. (1965). Fuzzy Sets - Information and Control, vol. 8, pp 338-353;
ZAPOROZEC, A. 2001. Contaminant source inventory. In: Zaporozec, A. (ed.) Groundwater contamination inventory. A methodological guideline. Paris, França: UNESCO.

ZILBERBRAND, M.; ROSENTHAL, E.; SHACHNAI, E. Impact of urbanization on hydrochemical evolution of groundwater and on unsaturated-zone gas composition in the coastal city of Tel Aviv, Israel. Journal of Contaminant Hydrology. 50; 3-4: 175-208. 2001.

ZWAHLEN, F. Vulnerability and risk mapping for the protection of carbonate (karst) aquifers, final report (COST action 620). European Commission, Directorate XII Science, Research and Development, Report EUR 20912, Brussels (2004) 297 pp. 